\title{
Use of Polyethylene Glycol Coatings for Optical Fibre Humidity Sensing
}

\author{
Sabriye Acikgoz, Bukem BiLen, Mustafa Muamer DemiR ${ }^{1}$, Yusuf Ziya Menceloglu², \\ Yani SKarlatos, Gulen AKTAS, and Mehmet Naci INCI* \\ Department of Physics, Bogazici University, Bebek 34342, Istanbul, Turkey \\ ${ }^{1}$ Department of Chemistry, Izmir Institute of Technology, Urla 35430, Izmir, Turkey \\ ${ }^{2}$ Faculty of Engineering and Natural Sciences, Sabanci University, Tuzla 34956, Istanbul, Turkey
}

(Received August 27, 2007; Accepted December 17, 2007)

\begin{abstract}
Humidity induced change in the refractive index and thickness of the polyethylene glycol (PEG) coatings are in situ investigated for a range from 10 to $95 \%$, using an optical waveguide spectroscopic technique. It is experimentally demonstrated that, upon humidity change, the optical and swelling characteristics of the PEG coatings can be employed to build a plastic fibre optic humidity sensor. The sensing mechanism is based on the humidity induced change in the refractive index of the PEG film, which is directly coated onto a polished segment of a plastic optical fibre with dipcoating method. It is observed that PEG, which is a highly hydrophilic material, shows no monotonic linear response to humidity but gives different characteristics for various ranges of humidity levels both in index of refraction and in thickness. It undergoes a physical phase change from a semi-crystalline structure to a gel one at around $80 \%$ relative humidity. At this phase change point, a drastic decrease occurs in the index of refraction as well as a drastic increase in the swelling of the PEG film. In addition, PEG coatings are hydrogenated in a vacuum chamber. It is observed that the hydrogen has a preventing effect on the humidity induced phase change in PEG coatings. Finally, the possibility of using PEG coatings in construction of a real plastic fibre optic humidity sensor is discussed.

(C) 2008 The Optical Society of Japan
\end{abstract}

Key words: humidity, sensors, plastic fibre, optics, polyethylene glycol

\section{Introduction}

The level of relative humidity has a vital role in our life and has unavoidable positive and negative effects on our environment, food and industrial products and all kinds of machinery. For example, an optimum level of relative humidity (RH) of about $40-60 \%$ is required to provide a healthy and comfortable environment for humans, while we need over $85 \%$ of $\mathrm{RH}$ in our refrigerators for food, vegetables and fruits to protect them against rotting and to preserve their freshness for a long time. Therefore, there is a need for a humidity control mechanism that enables one to maintain a desired humidity level. This has motivated a large number of researchers to propose and demonstrate various kinds of optical and non-optical humidity sensors, employing different operating concepts and test materials. ${ }^{1-13)}$

Bownass et al. ${ }^{14)}$ successfully demonstrated that polyethylene oxide can be used to detect humidity in passive optical networks at around the single value of $80 \% \mathrm{RH}$ for optical telecommunication wavelengths between 1.1 and $1.55 \mu \mathrm{m}$. The sensing mechanism is based on the humidityinduced change in the refractive index of the polyethylene oxide overlay on a polished surface of a half-block singlemode fibre device. Polyethylene oxide or poly(ethylene glycol) (PEG) is known to be highly hydrophilic in its response to water vapour. This makes PEG a favourite material for humidity sensing applications. However, to our knowledge, response mechanism of PEG thin films for a wide range of humidity - say, from 10 to $95 \%$ - together with their

${ }^{*}$ Corresponding author. E-mail address: naci.inci@boun.edu.tr humidity induced optical and swelling properties have not been investigated in detail.

In this paper, for the purpose of building a plastic optical fibre humidity sensor, the index of refraction and swelling behaviour of a PEG thin film are in situ determined using an optical waveguide spectroscopic method. It is experimentally observed that PEG has four distinct regions for humidity versus index of refraction and thickness. The PEG film undergoes a phase change at around $80 \%$ relative humidity, from a semi-crystalline state to a gel state. Changes in the refractive index and swelling are very abrupt at this specific humidity level. In this work, it is experimentally demonstrated that such optical and swelling data of the PEG coatings, particularly the phase change, can be modified if the PEG coating is enriched with hydrogen bonding under vacuum; and such a film can be used to construct a fibre optic humidity sensor for the humidity range from 15 to $90 \%$. The PEG coating is used as an overlay material on the polished surface of a poly(methyl methacrylate) (PMMA) plastic optical fibre. Our results show that the sensor output gives a fairly linear, reversible, fast and a repeatable response to humidity. The experimental results presented in this work suggest a practical motivation to investigate inexpensive PEG-based fibre optic sensors with a simple production mechanism and a simple principle of operation for relative humidity control; for example, in household appliances. Such a low-price and simple fibre optic sensor does not increase the cost of the white goods while contributing to their value-added prices and may have potential for multi-purpose use in various kinds of hightechnology electronic equipment. 


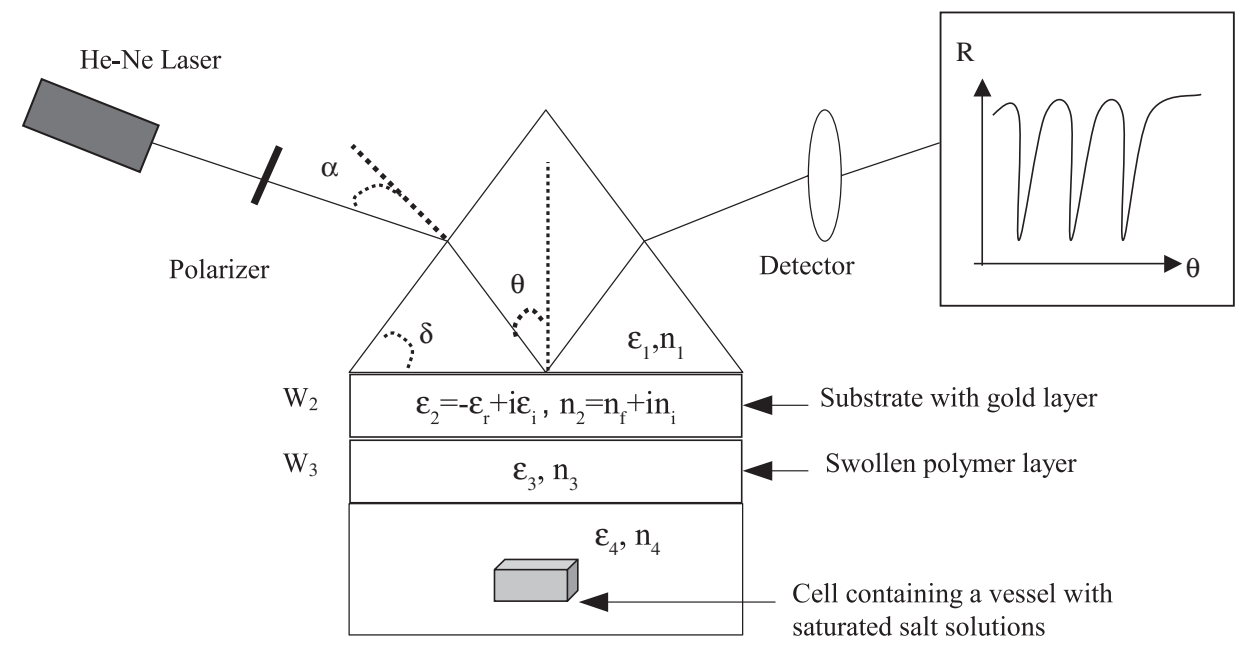

Fig. 1. Optical waveguide spectroscopy measurement setup.

\section{Experimental}

The optical waveguide spectroscopic setup (or, the Kretschmann ${ }^{15)}$ configuration of the attenuated total internal refection method) shown in Fig. 1 is employed to in situ determine the humidity induced changes in the index of refraction and thickness of a thin PEG polymer film. ${ }^{16)}$ The reflection of light as a function of the incident angle $\theta$ for a p-polarized monochromatic light beam of wavelength $\lambda$, for a four-layer system shown in Fig. 1, is given by ${ }^{17)}$

$$
\begin{gathered}
R(\theta)=\left|r_{1,4}(\theta)\right|^{2} \\
r_{t, 4}(\theta)=\frac{r_{t, t+1}(\theta)+r_{t+1,4}(\theta) \exp \left[2 i W_{t+1} k_{z t+1}(\theta)\right]}{1+r_{t, t+1}(\theta) r_{t+1,4}(\theta) \exp \left[2 i W_{t+1} k_{z t+1}(\theta)\right]}
\end{gathered}
$$

where $t=1,2$ and

$$
\begin{aligned}
r_{t, t+1}(\theta) & =\frac{\zeta_{t+1}(\theta)-\zeta_{t}(\theta)}{\zeta_{t+1}(\theta)+\zeta_{t}(\theta)} \quad(t=1,2,3) \\
k_{z t}(\theta) & =\frac{\omega}{c} \sqrt{\varepsilon_{t}-\left(\sqrt{\varepsilon_{1}} \sin \theta\right)^{2}} \\
\zeta_{t}(\theta) & =\frac{\varepsilon_{t}}{k_{z t}(\theta)} \quad(t=1,2,3,4)
\end{aligned}
$$

where $\omega$ and $c$ are the angular frequency and the velocity of light in vacuum, respectively, $k_{z t}$ is the wavevector component perpendicular to the interface in medium $t(t=1,2,3$ or 4$), r_{t, t+1}$ represents the refection ratio for the $t-(t+1)$ interface, $\varepsilon_{t}(t=1,2,3$ or 4$)$ is the dielectric constant of the media, $W_{t}(t=2$ or 3$)$ represents the thickness of the gold and PEG film.

Using the minima of experimental reflection spectra [i.e., $\alpha_{m}$ values at minima of $R(\theta)$ ], the index of refraction and thickness of the PEG film, which are shown in Fig. 1, represented by $n_{3}$ and $W_{3}$ respectively, are determined from the following equations: ${ }^{38)}$

$$
k W_{3}\left(n_{3}^{2}-N_{m}^{2}\right)^{1 / 2}=\psi_{m}\left(n_{3}, N_{m}\right)
$$

where

$$
N_{m}=\sin \alpha_{m} \cos \delta+\left(n_{1}^{2}-\sin ^{2} \alpha_{m}\right)^{1 / 2} \sin \delta
$$

and

$$
\begin{aligned}
& \psi_{m}\left(n_{3}, N_{m}\right)=m \pi+\Omega_{2}\left(n_{3}, N_{m}\right)+\Omega_{4}\left(n_{3}, N_{m}\right) \\
& \Omega_{j}\left(n_{3}, N_{m}\right)=\arctan \left[\left(\frac{n_{3}}{n_{j}}\right)^{2 \rho}\left(\frac{N_{m}^{2}-n_{j}^{2}}{n_{3}^{2}-N_{m}^{2}}\right)\right]^{1 / 2}
\end{aligned}
$$

with $j=2,4 . \delta$ is the prism angle (see Fig. 1) and $\rho$ is the polarization state of the laser beam ( $\rho=0$ for s-polarization and $\rho=1$ for p-polarization). Inserting eq. (7) into eqs. (6), (8), and (9) yields unique solutions of $n_{3}$ and $W_{3}$.

During angular scans, the reflected intensity $R(\theta)$ is detected by a large area silicon detector as a function of incidence angle $\theta$. Experimental spectra curves on reflection are fitted with theory using eq. (1).

\subsection{Optical setup}

A p-polarized monochromatic light of wavelength $632.8 \mathrm{~nm}$ from a helium-neon ( $\mathrm{He}-\mathrm{Ne})$ laser is employed as the monitoring beam. As shown in Fig. 1, the p-polarized light is coupled into the PEG film through a glass prism and a $\sim 50 \mathrm{~nm}$ thick gold thin film. The glass prism is made of BK7 material with a refractive index of 1.5151. At the stage of sample preparation, the gold and PEG films are initially deposited on a half-cm thick separate BK7 glass. The other empty side of this gold-PEG coated BK7 glass is brought into contact with the BK7 prism. An index matching oil of 1.5167 (Cargille Laboratories, NJ) is inserted between the glass prism and coated BK7 glass in order to avoid unwanted reflections from discontinuous interfaces.

The same optical setup shown in Fig. 1 is used to in situ determine the swelling kinetics and the humidity induced changes in the refractive index and film thickness. The PEG sample is brought into contact with a cell, which is held at a constant temperature and a constant humidity level. A vessel with saturated salt solutions ${ }^{19)}$ is inserted into the cell to control the humidity inside. The high salt content of the solution reduces the vapour pressure of the water to a distinct value. During moisture exposure the change in optical and physical properties are monitored by measuring 

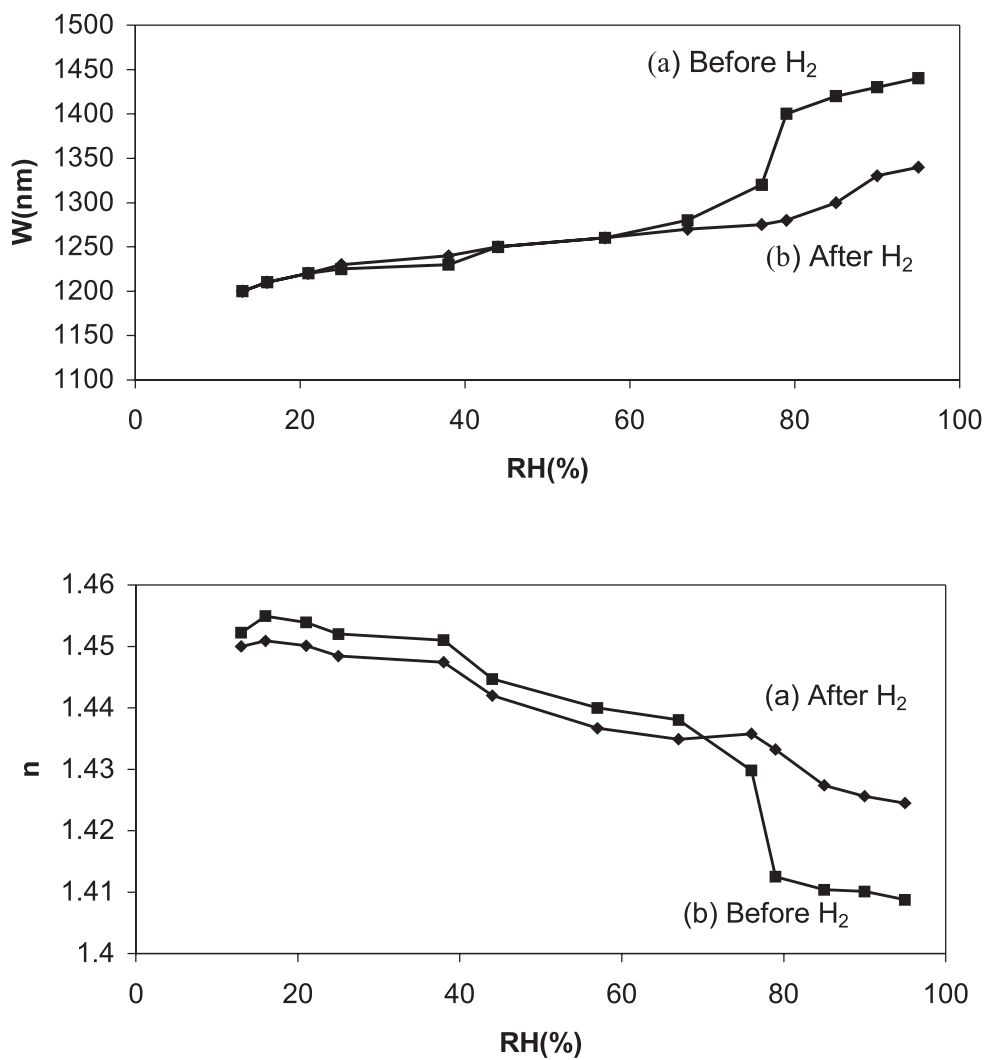

Fig. 2. Thickness as a function of the relative humidity of the environment before and after hydrogenation.
Fig. 3. Refractive index as a function of the relative humidity of the environment before and after hydrogenation. the shift in reflection spectra's minima of the waveguide modes. When a specific humidity value is reached - without changing the temperature of the vicinity - the sample is scanned as a function of the incident angle from $30^{\circ}$ to $90^{\circ}$ to obtain reflection spectra.

\subsection{Results}

Figure 2 shows the in situ relative humidity induced change in the thickness of pure and hydrogenated-PEG sample. It is clearly seen in curve (a) of Fig. 2 that as relative humidity of the environment increases the thickness of the pure PEG polymer increases due to absorption of water and the consequent swelling of the polymer film. Much to our surprise, an unexpected and distinctive swelling is observed in our experiments at a relative humidity around $80 \%$, which is indication of a phase change from one physical state to another. ${ }^{20)}$

Figure 3 shows in situ relative humidity induced change in the refractive index of pure and hydrogenated-PEG sample. As pure PEG sample is concerned, at very low humidity levels, between 13 and $16 \%$, there is a slight increase in the refractive index, from 1.452 to 1.455 . For this particular relative humidity region, $d n / d H_{\mathrm{R}}=+9.0 \times 10^{-4}$. As the water molecules start to diffuse into the PEG polymer layer, the refractive index begins to decrease from 1.455 to 1.430 for the relative humidity range from 16 to $76 \%$, at a relatively moderate level corresponding to $d n / d H_{\mathrm{R}}=$ $-4.0 \times 10^{-4}$. As the humidity reaches around $80 \%$, the refractive index of the PEG layer undergoes an abrupt decrease in a similar manner to that observed in the physical thickness of the polymer film shown in Fig. 2. $n$ decreases from 1.430 to 1.413 just for a small relative humidity range of about $3 \%$, from 76 to $79 \%$. This also confirms the phase change from one physical state to another. For this particular phase transition region $d n / d H_{\mathrm{R}}=-5.8 \times 10^{-3}$.

We should note that the humidity induced change in the refractive index is positive at very low humidity levels between 13 and 16\%, and negative beyond. In fact, for the pure PEG sample, due to the phase change process, it is clearly seen in Fig. 3 that $d n / d H_{\mathrm{R}}$ becomes most negative at humidity levels between 76 and $79 \%$. Moreover, the swelling ratio, $d W / W d H_{\mathrm{R}}$, between these humidity levels $(76-79 \%)$ is maximum.

The phase transition at a relative humidity of around $80 \%$ causes an abrupt change in the refractive index and thickness of the polymer layer. As stated above, this distinctive swelling occurs as a result of a transition between a semicrystalline state to a gel state of the polymer. Under increasing humidity, a critical activity of water is required to induce melting of the crystalline domains in the semicrystalline PEG. Once this humidity level is reached, an abrupt increase in swelling is expected. This transition occurs at a certain relative humidity level, known as the deliquescence relative humidity (DRH) ${ }^{21)}$ producing a saturated solution. More abrupt transitions in swelling and shrinking have been observed in spin coated polymers, ${ }^{22)}$ since covalent attachment of PEG layer suppress crystallization of the semi-crystalline polymer. Reduced crystallinity in covalently attached PEG would permit greater swelling in the low humidity region and, thus, a less abrupt transition.

At very low humidity levels, between 13 and $16 \%$, there is an increase in the refractive index. This might be a result of 
water extraction, which is already trapped inside the polymer. In this case, at very low humidity levels around $13 \%$, PEG chains contain a negligible amount of water. Since the refractive index of water (1.33) is lower than that of the PEG polymer $(\sim 1.47),{ }^{23)}$ as $\mathrm{H}_{2} \mathrm{O}$ molecules diffuse out of the PEG brushes from 13 to $16 \%$ humidity levels, an increase in the effective index of refraction $(n)$, which is a combination of both $n_{\mathrm{H}_{2} \mathrm{O}}$ and $n_{\mathrm{PEG}}$, is observed (see Fig. 3). However, beyond $16 \%$, chains cannot sustain the water intake and swelling starts; hence $n$ starts to decrease.

In the context of PEG coatings being applied as optical sensing material, one might envisage that the abrupt phase change at around $80 \%$ relative humidity, as seen in Figs. 2 and 3 , is a disadvantage in a pure PEG sample since a linear and monotonic output would be preferable over the present characteristics if the phase change from the semi-crystalline state to the gel state somehow can be prevented; or may be translated to even a further relative humidity beyond $80 \%$. We experimentally demonstrate here in this work that the phase change can be modified if the PEG coatings are enriched with hydrogen molecules. To verify this idea, the PEG sample is exposed to hydrogen gas in a vacuum chamber prior to optical waveguiding experiments. The hydrogen atoms form bonds with the ether oxygen atoms of the PEG chains. ${ }^{24)}$ Therefore, fewer ether oxygen atoms are available for the water molecules to make hydrogen bonding during the exposition of water vapour. The decreased interaction of the polymer with the water molecules decreases the response to humidity and the polymer has a more stable structure than before. To attain the phase change, the number of water molecules has to be increased, i.e., the phase change is observed at a higher humidity level (see the hydrogenated case in Figs. 2 and 3). Our measurements on hydrogenated PEG samples show that the hydrogenation of PEG films causes a less pronounced phase change from a semi-crystalline state to a gel state. As can be seen in Fig. 2, between 13 and 95\% relative humidity, the thickness increase of the PEG sample is less than before. The phase change is not abrupt and is shifted from around $80 \%$ to around $85 \%$ relative humidity. In Fig. 3, similarly after the hydrogenation process, it is observed that the refractive index of the PEG sample changes less and the phase change is gradual [see curve (a) in Fig. 3].

\section{PEG as a Fibre Optic Humidity Sensor}

Both pure PEG coatings and the hydrogenated ones presented in Figs. 2 and 3 - are used as overlay material for the replacement of the cladding-segment of a plastic optical fibre. A polymethylmethacrylate based plastic optical fibre (POF, Ck-30, Mitsubishi Rayon) is employed as a light transporter and a sensor substrate. A $2 \mathrm{~cm}$ segment of the $\mathrm{POF}$ at the centre is polished. Initial diameter of the POF is $750 \mu \mathrm{m}$ and it becomes about $735 \mu \mathrm{m}$ after polishing it. POF fibre is immersed into a PEG solution for dip-coating of the polymer film. To make the PEG coatings robust against its dissolution in water vapour or its removal from the fibre surface due to swelling at high relative humidity levels, a PEG polymeric solution is prepared following the procedure

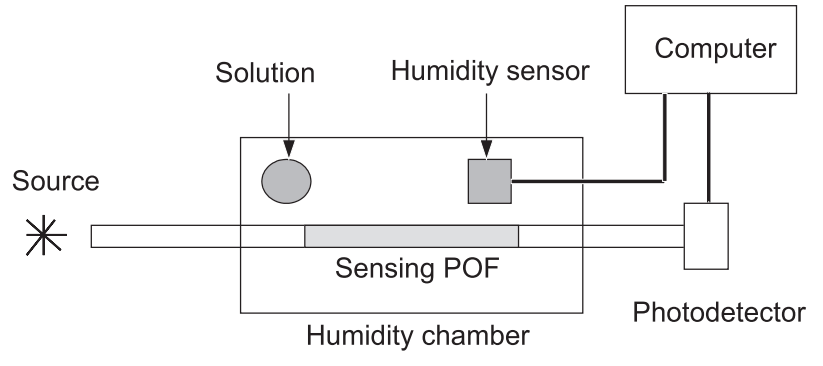

Fig. 4. Fibre optic experimental setup.

explained in previous studies. ${ }^{25,26)} \mathrm{POF}$ remains stationary and the polymer solution is allowed to flow with a speed of $0.25 \mathrm{~s} / \mathrm{ml}$. The coated POF is dried and kept in an oven at $50{ }^{\circ} \mathrm{C}$ for $4 \mathrm{~h}$ to crosslink the thin film on the polished segment of the POF.

The PMMA plastic optical fibre containing the optical sensing segment is located in a temperature controlled humidity box, as shown in Fig. 4. For the purpose of controlling the relative humidity, saturated salt solutions of $\mathrm{KNO}_{3}$ and $\mathrm{ZnCl}_{2}$ are inserted into the humidity chamber. $\mathrm{KNO}_{3}$ increases the relative humidity up to $95 \%$ at $295 \mathrm{~K}$, while $\mathrm{ZnCl}_{2}$ decreases it to about $13 \%$ at $296 \mathrm{~K}$.

The POF fibre has a core refractive index of 1.49 , numerical aperture of 0.5 , core diameter of $735 \mu \mathrm{m}$, and cladding diameter of $750 \mu \mathrm{m}$. This optical setup is kept simple on purpose for the sake of an inexpensive cost and an easy manufacturing procedure for mass production. This is why a POF of $750 \mu \mathrm{m}$ in diameter is used both as addressing fibre and as sensor substrate to avoid any extra launching or collection optics; for example, converging lenses, special holders, etc. Due to the same cost reduction policy, instead of a laser diode, an infrared light emitting diode (LED) with a central wavelength of $960 \mathrm{~nm}$ is used as illuminating monochromatic incoherent source to monitor humidity measurements. A silicon photodiode is used to convert optical signal to electrical voltage by monitoring humidityinduced change in the optical intensity of the LED. The LED is driven by an electrical current source and its driving current is monitored as reference data; thus allows us to avoid the use of an optical beam-splitter at the input of the POF to monitor fluctuations in the LED's intensity.

As stated above, PEG is a highly hydrophilic material and is a very good candidate for humidity sensing applications. The outcome PEG overlay is experimentally tested in many cycles at various humidity conditions, ranging from $10 \%$ up to $95 \%$, and is found to be nonsoluble in high water vapours, robust and durable at the plastic optical fibre surface. However, as seen in Fig. 5, the response of the PEG to humidity is not linear, which contains a turning point at around $80 \% \mathrm{RH}$. This is due to humidity induced change in the refractive index profile of this material, particularly because of the phase change from the semi-crystalline state to the gel state at around $80 \%$ RH [as seen in curve (a) of Fig. 2 and curve (b) of Fig. 3]. As can be distinctly seen in Fig. 5 that the sensor output versus humidity curve first of all shows a linear 

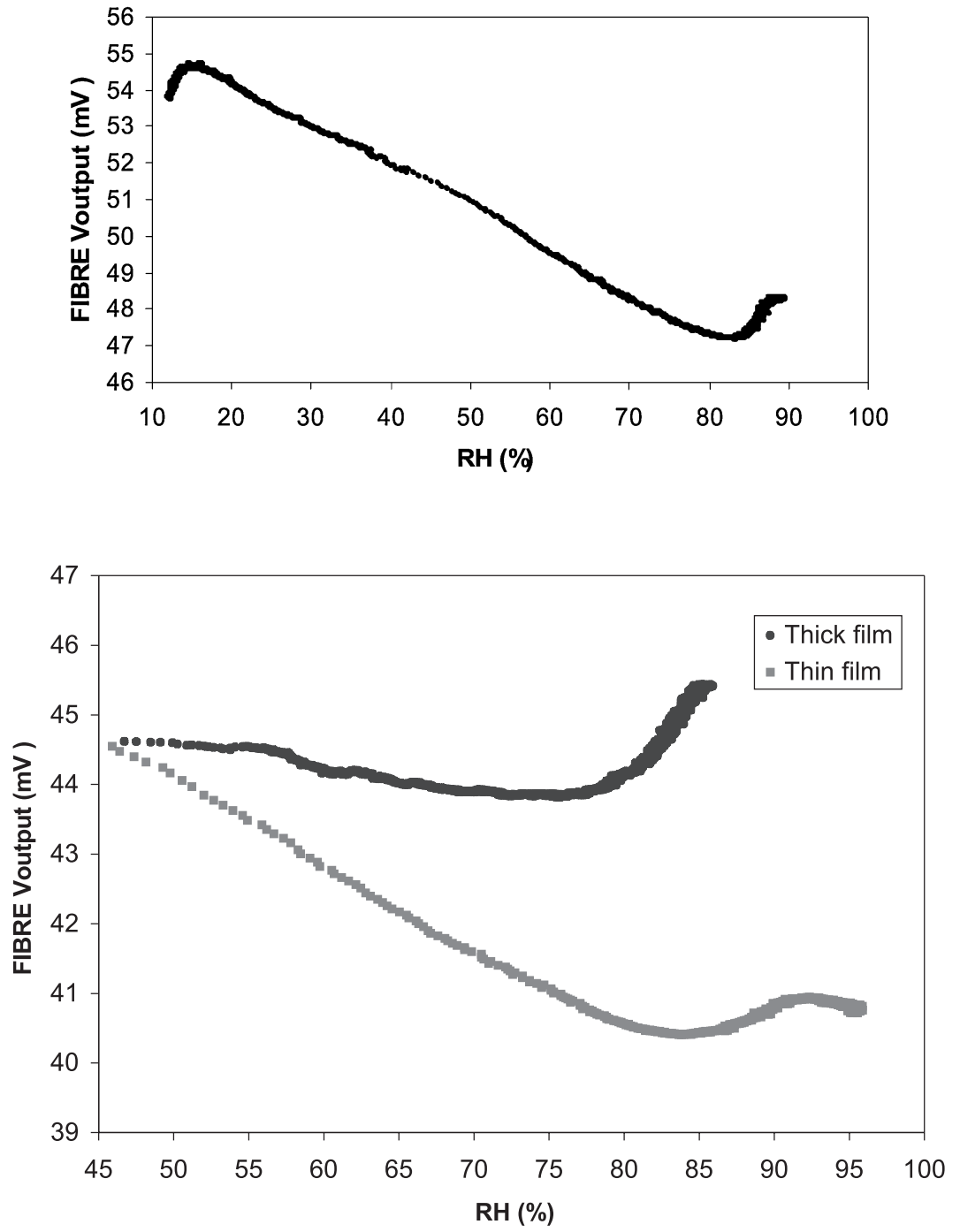

Fig. 5. PEG humidity sensor's behaviour versus humidity.

Fig. 6. The sensor response for different coating thickness. increase between 13 and $16 \%$, then shows a decrease between 16 and $79 \% \mathrm{RH}$ (at $23^{\circ} \mathrm{C}$ ); and then the sensor's response demonstrates a turning point at around $80 \% \mathrm{RH}$. A relatively steeper increase in the detected signal is recorded between 70 and $95 \% \mathrm{RH}$. In addition to this, the sensor response demonstrates a fading effect for thicker PEG overlays as the humidity decreases from 95 to $10 \% \mathrm{RH}$ (see Fig. 6). The cause of the later behaviour is rather simple to explain because it is clear that the PEG polymer network structure does not release humidity easily. In other words, the molecules of water vapour build up in the PEG network and hence the polymer brushes become incapable in evacuating the entire humidity. Thus causes the slope of the humidity curves (between 16 and $75 \%$ ) to approach to zero, as seen in Fig. 6.

The occurrence of the turning point in the humidity versus optical intensity at around $80 \% \mathrm{RH}$ for pure PEG coatings is quite noticeable as seen in Fig. 5. The mechanism behind this effect lies on the humidity caused decrease in the refractive index and swelling of the polymer film. With various possible overlay materials, the refractive index decreases as the humidity increases due to the increased water content in the film. Materials which exhibit this behaviour include gelatine, polyethylene oxide, polyvinyl alcohol as well as many other polymers. The change in optical properties with humidity can be seen from the following relation for the refractive index $n$ of a PEG-water composite material ${ }^{27)}$

$$
\frac{n^{2}-1}{n^{2}+2}=\sum_{i}\left(\frac{\rho_{i} R_{i}}{M_{i}}\right),
$$

where $\rho_{i}$ is density, $M_{i}$ is molecular weight, and $R_{i}$ is defined as the molar refractivity of the $i$ th component material. A change in any of the three parameters will change the refractive index of the film; an increase in water content of the film will cause it to swell slightly, resulting in a reduction in the overall density and hence a corresponding drop in refractive index.

When pure PEG is exposed to water vapor, hydrogen atoms of the water molecules make bonds with the ether oxygen atoms in the PEG chain. ${ }^{24}$ The increase in the amount of the absorbed water molecules causes PEG to swell; thus the density $\rho_{i}$ of the PEG is reduced, which causes a drop in $n$ of the PEG overlay coating, and a turning point in the humidity versus intensity curve is observed. This unusual characteristic of the swelling is not suitable 

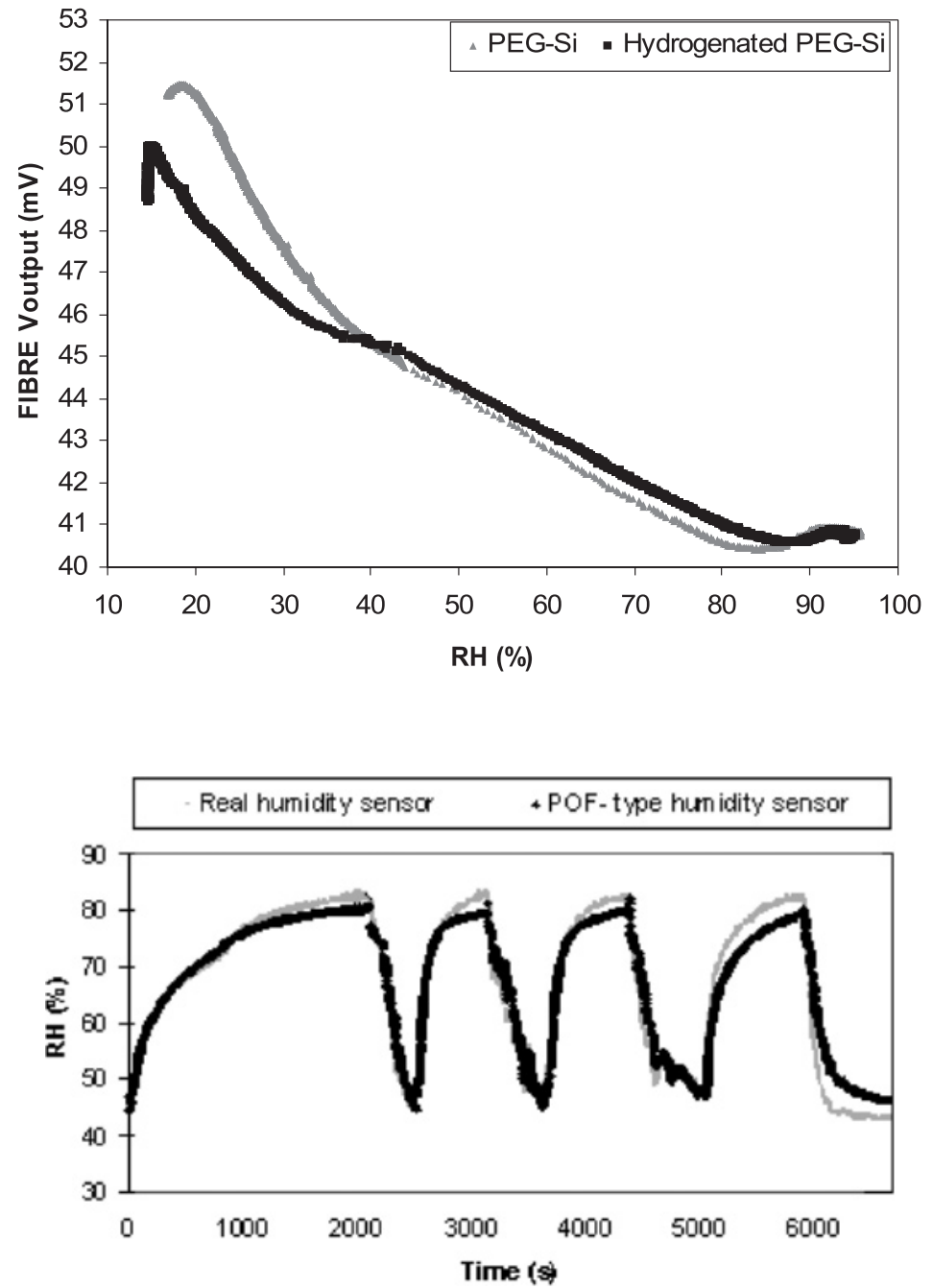

Fig. 7. Response of the hydrogenated PEG to humidity.
Fig. 8. Hydrogenated POF-type sensor and an electronic humidity sensor outputs. for a reliable humidity sensor. To increase the range of the $\mathrm{RH}$ detection with POF sensing technique and overcome the turning point in the intensity versus humidity curve in Fig. 5, the PEG layer coated on the fibre's polished segment is enriched with hydrogen under vacuum. After hydrogenation process, hydrogen atoms form bonds with the ether oxygen atoms of the polymer; therefore, the number of ether oxygen that can bind with water molecules decreases and a stable PEG network is obtained. Consequently, it is expected that the turning point occurring at the higher humidity values should disappear and the sensor humidity response should become fairly linear at the expense of a reduced sensitivity.

For the hydrogenation process, pure PEG coated samples are first heated in a vacuum chamber to $323 \mathrm{~K}$ for $2 \mathrm{~h}$ and at a pressure of $10^{-4} \mathrm{~Pa}$. After annealing the samples, the chamber is filled with hydrogen gas to a pressure of $10 \mathrm{~Pa}$, and they are kept in it for another $80 \mathrm{~min}$. In order to study the response characteristics of the hydrogenated sensor, measurements are carried out for different relative humidity values in the chamber. The humidity response of the thin film coated POF sensor is shown in Fig. 7. It should be noted that the sensor response becomes almost linear between 14 and $89 \%$ relative humidity values. After hydrogenation, the PEG film becomes less hydrophilic and absorbs less water molecules. Therefore, it starts to swell at higher humidity value. In this POF type sensor, swelling starts at $89 \% \mathrm{RH}$ value after the sample is enriched with hydrogen.

Figure 8 shows the response of the hydrogenated PEG coated POF for repeated cycles of humidity between 45 and $80 \%$. A commercially available electronic humidity sensor (Honeywell, IH-3610-1) is placed nearby the fibre optic sensor to compare their responses. It is clearly seen that the sensor successfully follows the changes in humidity with a reliable and quick response. We could not measure the response time of the POF sensor since the electronics used is not designed for this purpose.

\section{Discussion}

As humidity of the environment is increased, water molecules enter the PEG matrix and form hydrogen bonds with the oxygen atoms. When all oxygen atoms in the PEG polymer are occupied, additional water molecules penetrating from the outside into the PEG matrix form clusters with those previously absorbed water molecules. Such a cluster formation in the polymer causes a distinctive swelling and a phase change from a semi-crystalline state to a gel state. In our experiments, it is observed that the phase change in the thicker PEG coatings slightly shifted to lower humidity (see 
Fig. 6). This might be due to the water molecules being penetrated into the thicker polymer networks rather easily and causing a gel formation more quickly. This is why a thicker film shows rapid swelling response at lower $\mathrm{RH}$ values - around $75 \%$ - as seen in Fig. 6. In addition, after gel formation in a thicker film, there is a steeper increase in the output intensity of the fibre sensor. For thicker films, there is more space for the water molecules to build up inside the PEG network and a further reduction in the overall density due to an increase in the water content causes a further decrease in the refractive index, and therefore a further increase in the intensity. Besides, releasing out these trapped water molecules from the polymer network is more difficult and sometimes impossible. As a result, thin PEG coatings are more suitable as sensing overlays.

When PEG samples are exposed to hydrogen gas, hydrogen atoms are bound to polymer's oxygen atoms; therefore, fewer oxygen atoms become available for the water molecules to make hydrogen bonding during exposure to water. The decreased interaction of the polymer with water molecules decreases the response to humidity. In other words, the polymer absorbs less water molecules and hence it becomes more robust against the phase change upon swelling. This stability in the PEG structure prevents swelling to start at lower humidity levels; it shits the swelling point to a higher humidity level - around $95 \%$ (see Fig. 7). Besides, our experiments show that the hydrogenation is ineffective for thick PEG films. When a thick polymer film coated fibre is exposed to hydrogen gas, the number of ether oxygen atoms decreases. However, this reduction is not sufficient to prevent diffusion of the water molecules into the PEG network and to shift the turning point to higher RH values.

\section{Conclusion}

Refractive index and thickness of poly(ethylene glycol) (PEG) coatings upon humidity changes are in situ studied in details. These coatings are coated onto a polished-segment of a plastic optical fibre for the purpose of humidity sensing applications. The swelling process and the phase transition in thin PEG films are observed to be reversible. It is experimentally observed that the linear characteristics, reversibility and sensitivity of hydrogenated-PEG coatings to humidity range between 14 and 95\% RH make PEG a suitable material for humidity sensing applications.

\section{Acknowledgement}

This work was supported by The Scientific and Technological Research Council of Turkey and Bogazici University Research Fund, under contract numbers $107 \mathrm{~T} 206$ and 05HB301, respectively.

\section{References}

1) S. K. Shukla, G. K. Parashar, A. P. Mishra, P. Misra, B. C Yadav, R. K. Shukla, L. M. Bali, and G. C. Dubey: Sens. Actuators B 98 (2004) 5.

2) S. Muto, O. Suzuki, T. Amano, and M. Morisawa: Meas. Sci. Technol. 14 (2003) 746.

3) P. Kronenberg, P. K. Rastogi, P. Giaccari, and H. G. Limberger: Opt. Lett. 27 (2002) 1385.

4) Z. M. Rittersma: Sens. Actuators A 96 (2002) 196.

5) R. Jindal, S. O. Tao, J. P. Singh, and P. S. Gaikwad: Opt. Eng. 41 (2002) 1093.

6) S. J. Glenn, B. M. Cullum, R. B. Nair, D. A. Nivens, C. J. Murphy, and S. M. Angel: Anal. Chim. Acta 448 (2001) 1.

7) S. McMurtry, J. D. Wright, and D. A. Jackson: Sens. Actuators B 67 (2000) 52.

8) F. J. Arregui, Y. J. Liu, I. R. Matias, and R. O. Claus: Sens. Actuators B 59 (1999) 54.

9) B. D. Gupta and Ratnanjali: Sens. Actuators B 80 (2001) 132.

10) G. H. Cross, Y. T. Ren, and N. J. Freeman: J. Appl. Phys. 86 (1999) 6483.

11) S. Otsuki, K. Adachi, and T. Taguchi: Anal. Sci. 14 (1998) 633.

12) Z. A. Ansari, R. N. Karekar, and R. C. Aiyer: Thin Solid Films 305 (1997) 330.

13) M. N. Weiss, R. Srivastava, and H. Groger: Electron. Lett. 32 (1996) 842.

14) D. C. Bownass, J. S. Barton, and J. D. C. Jones: Opt. Lett. 22 (1997) 346.

15) F. S. Damos, R. C. S. Luz, and L. T. Kubota: Langmuir 21 (2005) 602.

16) B. Bilen: Ph. D. Thesis, Faculty of Arts and Sciences, Bogazici University, 2007.

17) E. Kretschmann: Z. Phys. 241 (2005) 313.

18) R. Ulrich and R. Torge: Appl. Opt. 12 (1973) 2901.

19) M. Biesalski and J. Rühe: Langmuir 16 (2000) 1943.

20) W. L. Chen, K. R. Shull, T. Papatheodorou, D. A. Styrkas, and J. L. Keddie: Macromolecules 32 (1999) 136.

21) J. H. Seinfeld and S. N. Pandis: Atmospheric Chemistry and Physics (Wiley/VCH, Weinheim, 1998) Ch. 9, p. 507.

22) G. Tae, J. A. Kornfield, J. A. Hubbell, and D. Johannsmann: Langmuir 18 (2002) 8241.

23) T. M. Aminabhavi and K. Banerjee: J. Chem. Eng. Data 43 (1998) 852.

24) K. L. A. Chan and S. G. Kazarian: Vib. Spectrosc. 35 (2004) 45.

25) O. Erdamar, Y. Skarlatos, G. Aktas, and M. N. Inci: Appl. Phys. A 83 (2006) 159.

26) H. U. Yogun, Y. Ercil, Y. Menceloglu, and M. N. Inci: European Patent WO2006011117 (2006).

27) C. Ronot, H. Archenault, H. Gagnaire, J. P. Goure, N. Jaffrezic-Renault, and T. Pichery: Sens. Actuators B 11 (1993) 375 . 